Imaging molecular shapes with molecular-frame photoelectron angular distributions from core hole ionization

This article has been downloaded from IOPscience. Please scroll down to see the full text article.

2012 J. Phys. B: At. Mol. Opt. Phys. 45194002

(http://iopscience.iop.org/0953-4075/45/19/194002)

View the table of contents for this issue, or go to the journal homepage for more

Download details:

IP Address: 198.189.57.10

The article was downloaded on 28/09/2012 at 23:14

Please note that terms and conditions apply. 


\title{
Imaging molecular shapes with molecular-frame photoelectron angular distributions from core hole ionization
}

\author{
C S Trevisan ${ }^{1}$, C W McCurdy ${ }^{2,3}$ and T N Rescigno ${ }^{3}$ \\ ${ }^{1}$ Department of Sciences and Mathematics, California Maritime Academy, Vallejo, California 94590, \\ USA \\ ${ }^{2}$ Department of Chemistry, University of California, Davis,CA 95616, USA \\ ${ }^{3}$ Lawrence Berkeley National Laboratory, Chemical Sciences and Ultrafast X-ray Science Laboratory, \\ Berkeley, CA 94720, USA \\ E-mail: tnrescigno@lbl.gov
}

Received 8 March 2012, in final form 14 April 2012

Published 24 September 2012

Online at stacks.iop.org/JPhysB/45/194002

\begin{abstract}
We demonstrate, for a class of molecules containing a single heavy atom, the striking result that molecular-frame photoelectron angular distributions resulting from core-level ionization can be used to obtain three-dimensional images of the target molecule at low photoelectron energies. We demonstrate this finding with the results of theoretical calculations on methane, ammonia and water.
\end{abstract}

(Some figures may appear in colour only in the online journal)

\section{Introduction}

Conventional measurements of molecular photoelectron angular distributions are made in the laboratory frame, where the averaging over random orientations of the target with respect to the photon beam leads to the familiar $\left[1+\beta(E) P_{2} \cos ((\theta))\right]$ distribution, where $P_{2}$ is a Legendre polynomial and $\beta(E)$ is the energy-dependent asymmetry parameter. By way of contrast, photoelectron angular distributions, when viewed from the molecular frame, have a generally far richer structure that cannot be described by a single parameter $[1,2]$. To measure a molecular-frame photoelectron angular distribution (MFPAD), the molecular target must be oriented accurately in the laboratory frame. This can be done prior to ionization by laser alignment [3, 4]. Alternatively, the orientation can be deduced after core-level photoionization by detecting the photoelectron in coincidence with positively charged fragments [5] that emerge following the dissociation that accompanies prompt Auger decay [6, 7]. The latter technique was recently shown in the case of the methane molecule to be capable of providing striking threedimensional (3D) images of the isolated molecule caused by the focusing of photoelectrons along the bond directions $[6,7]$. Such images only emerge when the MFPADs are averaged over photon polarization directions.
In this study, we examine whether the MFPAD imaging we found for methane was specific to that molecule or whether similar imaging takes place in the case of other target molecules that contain a single heavy atom. We show the results of similar studies on ammonia and water and demonstrate that, over a specific range of photoelectron energies, the MFPADs faithfully image the target molecule. The fact that core-level MFPADs can be sensitive probes of molecular shapes may prove useful in the context of pumpprobe experiments with ultra-short x-ray probe pulses, which are expected to become available with the next generation of free-electron lasers. Since MFPADs depend on molecular geometry [8], the kind of imaging demonstrated here could then be taken into the time domain [9-11] to track conformational changes in a photo-excited molecule in real time [12].

In the following section we briefly review the computational method used to compute the photoelectron angular distribution. We then present results of computations on $\mathrm{CH}_{4}, \mathrm{NH}_{3}$ and $\mathrm{H}_{2} \mathrm{O}$. We conclude with a brief discussion.

\section{Theory}

Photoionization cross sections in the molecular frame can be constructed from the matrix elements

$$
I_{\Gamma_{0}}^{\mu}=\left\langle\Psi_{\Gamma_{0}}^{-}\left|r_{\mu}\right| \Psi_{0}\right\rangle
$$


where $r_{\mu}$ is a component of the dipole operator, which we evaluate here in the length form,

$$
r_{\mu}= \begin{cases}z, & \mu=0 \\ \mp(x \pm \mathrm{i} y) / \sqrt{2}, & \mu= \pm 1\end{cases}
$$

$\Psi_{0}$ is the initial state wavefunction of the $N$-electron target and $\Psi_{\Gamma_{0}}^{-}$is the final-state wavefunction for production of photoions in a specific cation state $\Gamma_{0}$. Note that we are using $\Gamma_{0}$ as a combined index to denote the target ion electronic state and the angular momentum quantum numbers $l_{0}, m_{0}$ of the ejected photoelectron. The final-state wavefunction is written as

$$
\Psi_{\Gamma_{0}}^{-}=\sum_{\Gamma} A\left(\chi_{\Gamma} F_{\Gamma \Gamma_{0}}^{-}\right)+\sum_{i} d_{i}^{\Gamma_{0}} \Theta_{i},
$$

where $\Gamma$ labels the final ionic target states $\chi_{\Gamma}$ included, $F_{\Gamma \Gamma_{0}}^{-}$ are channel functions that describe the photoionized electron, $A$ is the antisymmetrization operator and the $\Theta_{i}$ s are $N$ electron correlation terms. In the present application, only one ionic target state is included in the trial wavefunction, that being the core-hole state. We compute the final-state continuum wavefunction using the well-established complex Kohn variational method, which can be applied to electron scattering from polyatomic ions. Since the computational details of the Kohn method, including its application to polyatomic molecular photoionization [13], have been detailed elsewhere [14], those details will not be repeated here. In the Kohn method, the channel functions are expanded, in the molecular frame, as

$$
\begin{aligned}
& F_{\Gamma \Gamma_{0}}^{-}(\mathbf{r})=\sum_{i} c_{i}^{\Gamma \Gamma_{0}} \varphi_{i}(\mathbf{r})+\sum_{l m}\left[f_{l}\left(k_{\Gamma}, r\right) \delta_{l l_{0}} \delta_{m m_{0}} \delta_{\Gamma \Gamma_{0}}\right. \\
& \left.\quad+T_{l l_{0} m m_{0}}^{\Gamma \Gamma_{0}} h_{l}^{-}\left(k_{\Gamma}, r\right)\right] Y_{l m}(\hat{\mathbf{r}}) / k_{\Gamma}^{\frac{1}{2}} r
\end{aligned}
$$

where the $\varphi_{i}(\mathbf{r})$ are a set of square-integrable (Cartesian Gaussian) functions, $Y_{l m}$ is a normalized spherical harmonic, $k_{\Gamma}$ are channel momenta, and the $f_{l}\left(k_{\Gamma}, r\right)$ and $h_{l}^{-}\left(k_{\Gamma}, r\right)$ are numerical radial continuum functions that behave asymptotically as regular and incoming partial-wave Coulomb functions, respectively [15]. The coefficients $T_{l l_{0} m m_{0}}^{\Gamma \Gamma_{0}}$ are the $T$ matrix elements.

To avoid the practical difficulties of dealing with nonorthogonal orbitals, the bound initial state and final combined state of the photoelectron and the ion are constructed using a common set of orthogonal orbitals. Those orbitals are in turn chosen as the natural orbitals from the averaged density matrices of the ion and neutral molecules, effectively applying what is known as 'Slater's transition-state approximation' [16] for the photoionization process. The neutral initial state wavefunction, $\Psi_{0}$, is constructed as a single configuration from those natural orbitals. Slater's transition-state orbitals have been used extensively in core-level photoionization studies and have been shown to give results consistent with experimental measurements [17-20]. The complex Kohn scattering calculation then employs the static-exchange approximation with the target cation wavefunction constructed as a single configuration of the same natural orbitals.

In order to construct an amplitude that represents a photoelectron with momentum $k_{\Gamma_{0}}$ ejected by absorption of linearly polarized photons with polarization direction $\hat{\epsilon}$, measured relative to the molecular body-frame, the matrix elements $I_{\Gamma_{0}}^{\mu}$ must be combined in a partial wave series

$$
I_{\hat{k}, \Gamma_{0}, \hat{\epsilon}}=\sqrt{\frac{4 \pi}{3}} \sum_{\mu l_{0} m_{0}} \mathrm{i}^{-l_{0}} \mathrm{e}^{\mathrm{i} \delta_{l_{0}}} I_{\Gamma_{0}}^{\mu} Y_{1 \mu}(\hat{\epsilon}) Y_{l_{0} m_{0}}(\hat{k}),
$$

where $\delta_{l_{0}}$ is a Coulomb phase shift. The cross section (MFPAD), differential in the angle of photoejection and photon polarization relative to the fixed body-frame of the molecule, is then given by

$$
\frac{\mathrm{d}^{2} \sigma}{\mathrm{d} \Omega_{\hat{k}} d \Omega_{\hat{\epsilon}}}=\frac{8 \pi \omega}{3 c}\left|I_{\hat{k}, \Gamma_{0}, \hat{\epsilon}}\right|^{2},
$$

where $\omega$ is the photon energy and $c$ is the speed of light.

To calculate the polarization-averaged MFPAD, we must integrate the doubly differential cross section given in equation (6) over all $\Omega_{\hat{\epsilon}}$. This is obtained by substituting equation (5) into equation (6) and using the orthonormality of the spherical harmonics $Y_{1 \mu}(\hat{\epsilon})$ to obtain

$$
\begin{aligned}
& \int \frac{\mathrm{d}^{2} \sigma}{\mathrm{d} \Omega_{\hat{k}} \mathrm{~d} \Omega_{\hat{\epsilon}}} \mathrm{d} \Omega_{\hat{\epsilon}}=\frac{8 \pi \omega}{3 c} \frac{4 \pi}{3} \sum_{\mu}\left|\sum_{l_{0} m_{0}} I_{\Gamma_{0}}^{\mu} Y_{l_{0} m_{0}}(\hat{k})\right|^{2} \\
& \quad \equiv \sum_{\mu} M_{\mu}(\hat{k})
\end{aligned}
$$

Equation (7) is simply the incoherent sum of the MFPADs over the three components of the dipole operator. We should emphasize that, while this quantity does not depend on photon polarization, there is no a priori reason why it should image the shape of the molecule.

\section{Results}

The utility of the core-level MFPAD, integrated over all polarization directions, as a tool for imaging the molecule, is demonstrated by the $3 \mathrm{D}$ images shown in figures $1-3$ which show results for methane, ammonia and water, respectively. In all cases, the integrated MFPAD faithfully reproduces the shape of the target molecule for a range of photon energies that extends from near threshold to $\sim 10 \mathrm{eV}$ above the K-edge. We also note that at the highest energy shown, $27.21 \mathrm{eV}$, the integrated MFPADs, while they have to correct symmetry, i.e. $\mathrm{T}_{d}, \mathrm{C}_{3 v}$ and $\mathrm{C}_{2 v}$, respectively, no longer look like the molecule. In the high energy limit, the integrated MFPADs would all be approximately spherically symmetric.

In the energy range where the integrated MFPADs image the molecule, the underlying polarization-dependent MFPADs demonstrate a competition between two effects. The first is the evident focusing of the outgoing electrons in the directions of the bonds by the molecular potential. This is moderated by the second effect, which is the tendency of the photoelectron to follow the axis of polarization, which dominates at high energy. These effects are illustrated in figures 4-6, which show MFPADS at $E=4.35 \mathrm{eV}$ for the molecules in question for three different photon polarization directions. It is interesting to note the clear tendency of the photoelectron to focus along the bonds, except in those cases where a bond lies in the plane perpendicular to the axis of polarization, in which case, ejection along said bond vanishes by dipole selection rules. 

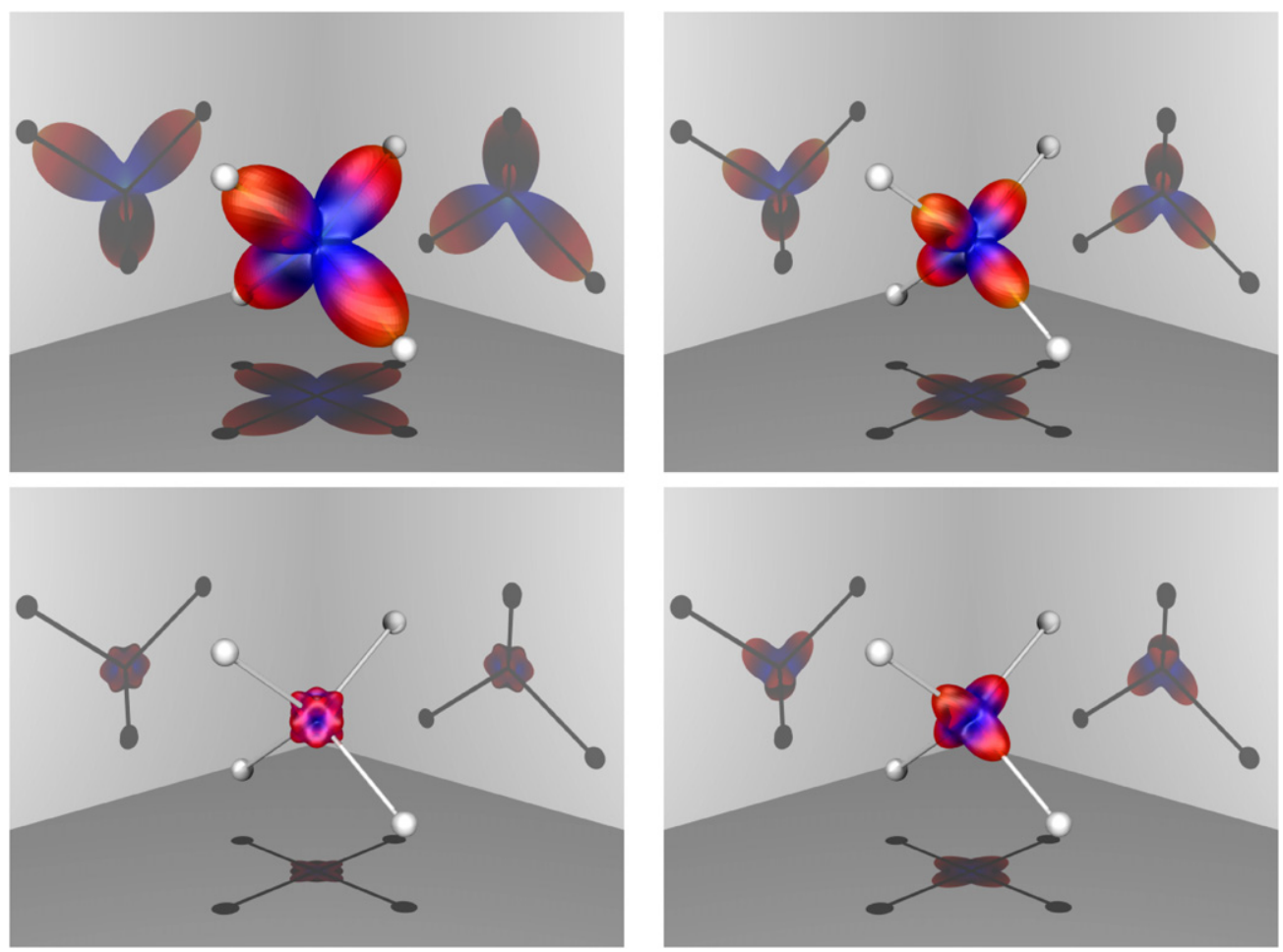

Figure 1. Integrated MFPADs for K-shell ionization in $\mathrm{CH}_{4}$ calculated using the complex Kohn variational method. Photoelectron energies, clockwise from upper left, are 1.09, 4.35, 9.80 and $27.21 \mathrm{eV}$, respectively. The MFPADs are plotted in arbitrary, but internormalized, units. The radial distance to each point on a plotted surface is proportional to the value of the cross section at that point, while the colour shading is merely a visual guide with magnitude increasing from blue to red.
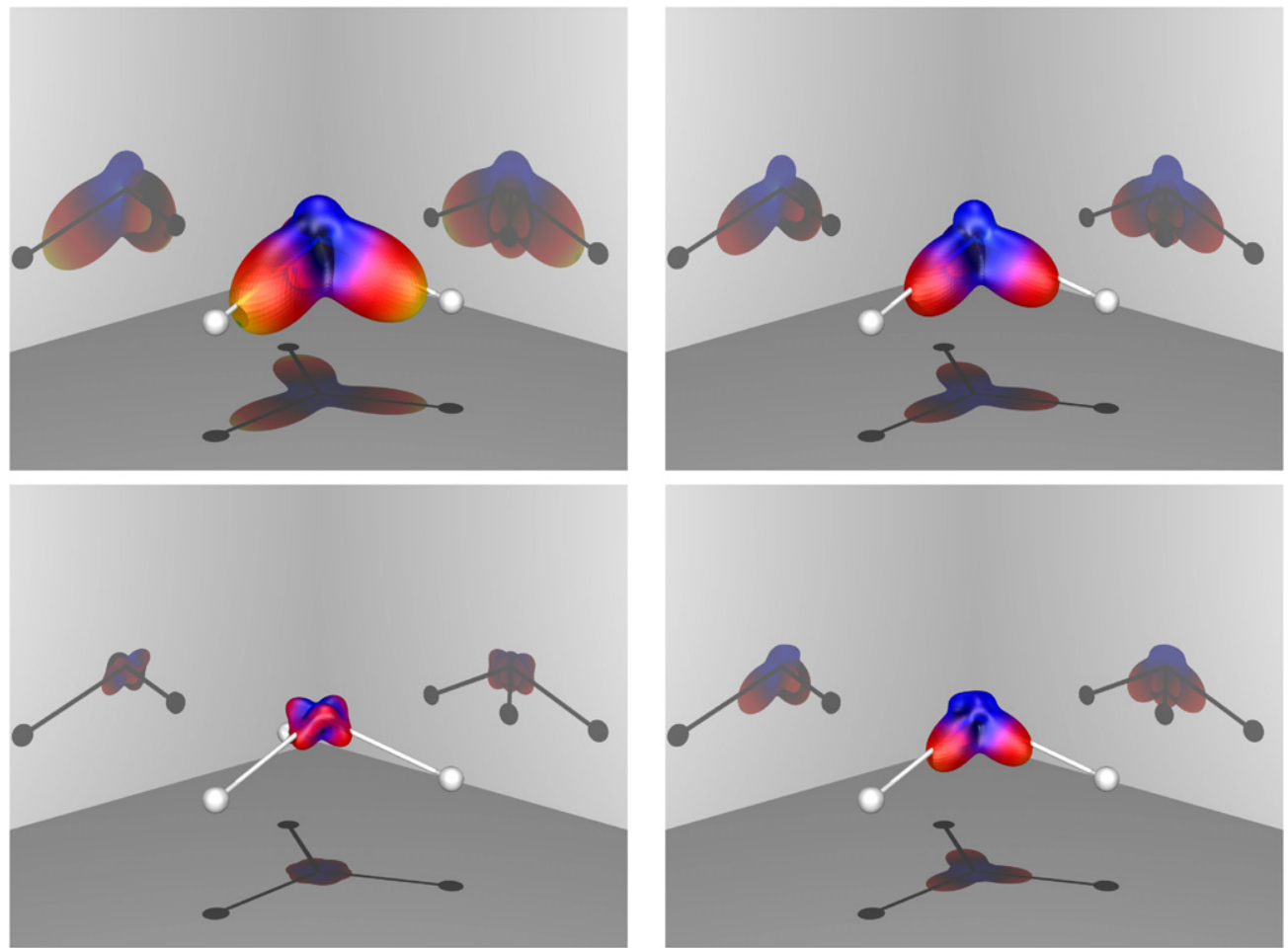

Figure 2. As in figure 1, for $\mathrm{NH}_{3}$.

\section{Discussion}

We have demonstrated that core-level MFPADs, when integrated over all polarization directions, can provide striking
3D images of small molecules composed of hydrogen and a single heavy atom over a range of photoelectron energies extending from threshold to $\sim 10 \mathrm{eV}$ photoelectron energy. To realize such images experimentally, the molecular target 

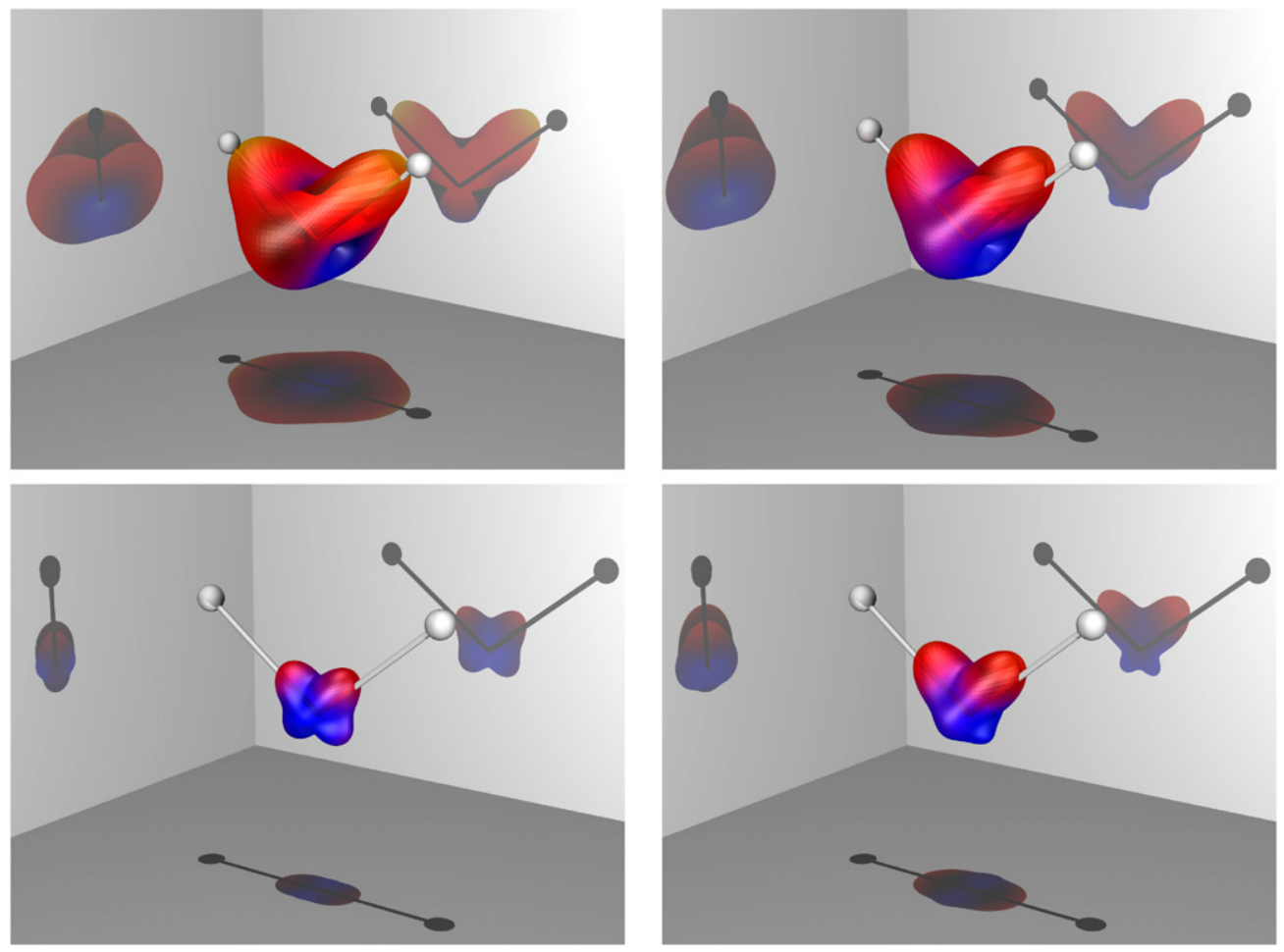

Figure 3. As in figure 1, for $\mathrm{H}_{2} \mathrm{O}$.
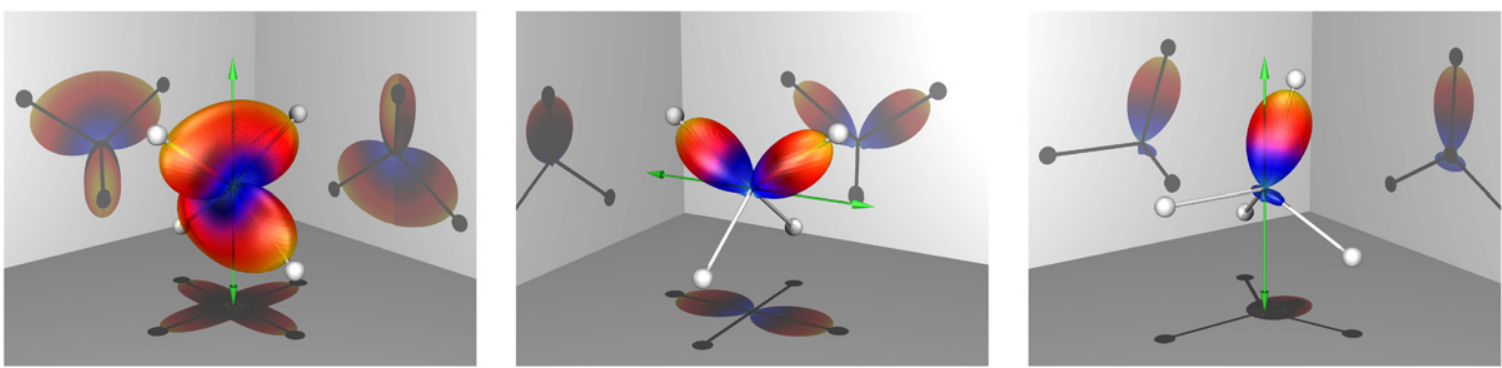

Figure 4. MFPADs for $\mathrm{K}$-shell ionization of $\mathrm{CH}_{4}$ calculated using the complex Kohn variational method at $4.35 \mathrm{eV}$. The axis of photon polarization is indicated by the double-ended arrow. The photon polarization lies along a $\mathrm{C}_{2}$ axis (left panel), perpendicular to a $\mathrm{C}_{2}$ axis (centre panel) and close to a $\mathrm{C}_{3}$ axis (right panel).
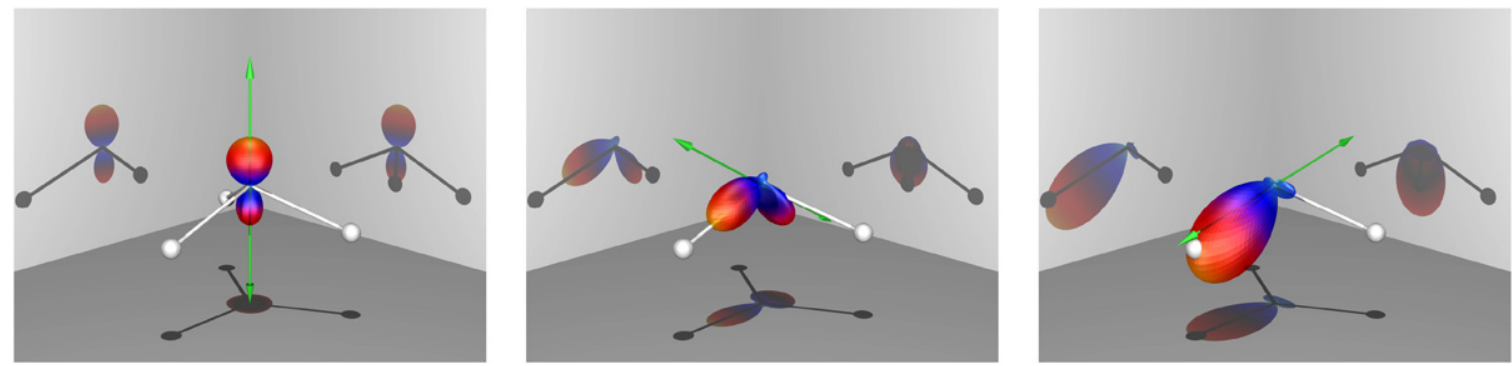

Figure 5. As in figure 4, for $\mathrm{NH}_{3}$. The polarization vectors are all chosen to lie in a plane defined by the $\mathrm{C}_{3}$ axis and one $\mathrm{N}-\mathrm{H}$ bond. The angle between the polarization vector and the $\mathrm{C}_{3}$ axis is $0^{\circ}$ (left panel), $60^{\circ}$ (centre panel) and $105^{\circ}$ (right panel).

must be oriented in the laboratory frame, either prior to photoabsorption by laser alignment, or subsequently by momentum imaging of photoelectrons and ion fragments measured in coincidence. The latter technique has already been realized in the case of $\mathrm{CH}_{4}[6,7]$, where core ionization opens a strong simultaneous double Auger decay channel that can dissociate promptly to three positively charged fragments. The detection of three charged fragments allows the complete orientation of the molecule to be determined. A similar direct double Auger fragmentation has been observed in water [21], and theoretical calculations we have carried out lead us to believe that this will also be the case in ammonia. 

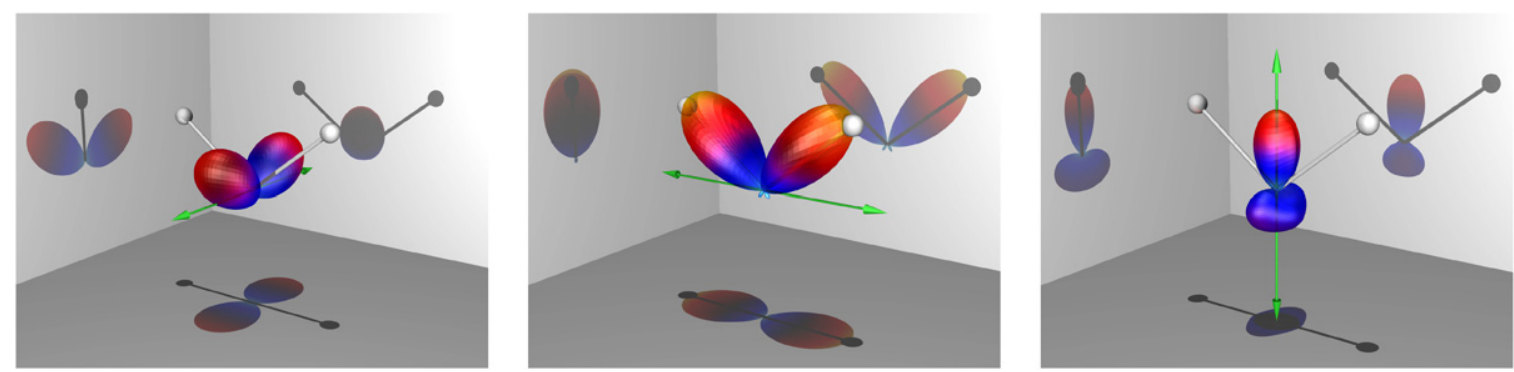

Figure 6. As in figure 4, for $\mathrm{H}_{2} \mathrm{O}$. The polarization vectors are chosen to lie along the $x$-axis (left panel), $y$-axis (centre panel) and $z$-axis (right panel), with the molecule in the $y z$-plane.

The simple picture presented here becomes more complicated in the case of molecules containing more than one heavy atom. In that case, a core-level electron ejected from a particular atomic site can be diffracted through multiple scattering from other heavy atomic centres to produce MFPADs that are more structured than a simple 3D image of the target [12]. Even in these cases, the MFPADs can be very sensitive to molecular geometry and offer the possibility of capturing time-dependent images of a photo-excited molecule with ultrafast pump-probe measurements [9-11].

\section{Acknowledgments}

This work was performed under the auspices of the US Department of Energy by LBNL under contract no. DE-AC02-05CH11231 and supported by the US DOE Office of Basic Energy Sciences, Division of Chemical Sciences. CST acknowledges support from a CSU Martime SoTL grant.

\section{References}

[1] Shigemasa E, Adachi J, Oura M and Yagishita A 1995 Phys. Rev. Lett. 74359

[2] Mizuno T, Adachi J, Miyauchi N, Kazama M, Stener M and Decleva P 2012 J. Chem. Phys. 136074305

[3] Larsen J J, Hald K, Bjerre N, Stapelfeldt H and Seideman T 2000 Phys. Rev. Lett. 852470

[4] Hansen J L et al 2011 Phys. Rev. A 83023406
[5] Dörner R, Mergel V, Jagutzki O, Spielberger L, Ullrich J, Moshammer R and Schmidt-Böcking H 2000 Phys. Rep. 33095

[6] Williams J B et al 2012 Phys. Rev. Lett. at press

[7] Williams J B et al 2012 J. Phys. B: At. Mol. Opt. Phys. 45194003

[8] Arasaki Y, Takatsuka K, Wang K and McKoy V 2010 J. Chem. Phys. 132124307

[9] Geßner O et al 2006 Science 311219

[10] Bisgaard C Z, Clarkin O J, Wu G, Lee A M D, Geßner O, Hayden C C and Stolow A 2006 Science 311219

[11] Hockett P, Bisgaard C Z, Clarkin O J and Stolow A 2011 Nature Phys. 7612

[12] Rescigno T N, Douguet N and Orel A E 2012 J. Phys. B: At. Mol. Opt. Phys. 45194001

[13] Rescigno T N, Lengsfield B H III and Orel A E 1993 J. Chem. Phys. 995097

[14] Rescigno T N, Lengsfield B H III and McCurdy C W 1995 Modern Electronic Structure Theory vol 1 ed D R Yarkony (Singapore: World Scientific) p 501

[15] Rescigno T N and Orel A E 1991 Phys. Rev. A 431625

[16] Slater J C 1974 The Self-Consistent Field for Molecules and Solids: Quantum Theory of Molecules and Solids vol 4 (New York: McGraw-Hill)

[17] Liu X J et al 2008 Phys. Rev. Lett. 101083001

[18] Saito N et al 2003 J. Phys. B: At. Mol. Opt. Phys. 36 L25

[19] Zähringer K, Meyer H D and Cederbaum L S 1992 Phys. Rev. A 45318

[20] Schirmer J, Braunstein M and McKoy V 1990 Phys. Rev. A $41283-300$

[21] Yamazaki M, Adachi J, Teramoto T, Yagishita A, Stener M and Decleva P 2009 J. Phys. B: At. Mol. Opt. Phys. 42051001 